CLINICAL RESEARCH ARTICLE

\title{
Acute kidney injury in critically III children and young adults with suspected SARS-CoV2 infection
}

Rajit K. Basu ${ }^{1 凶}$, Erica C. Bjornstad ${ }^{2}$, Katja M. Gist ${ }^{3}$, Michelle Starr ${ }^{4}$, Paras Khandhar ${ }^{5}$, Rahul Chanchlani ${ }^{6}$, Kelli A. Krallman ${ }^{7}$, Michael $^{2}$ Zappitelli ${ }^{8}$, David Askenazi ${ }^{2}$, Stuart L. Goldstein ${ }^{7}$ on behalf of the SPARC Investigators

(c) The Author(s), under exclusive licence to the International Pediatric Research Foundation, Inc 2021

BACKGROUND: We aimed to study the association of suspected versus confirmed infection with the novel SARS-CoV2 virus with the prevalence of acute kidney injury (AKI) in critically ill children.

METHODS: Sequential point-prevalence study of children and young adults aged 7 days to 25 years admitted to intensive care units under investigation for SARS-CoV2 infection. AKI was staged in the first 14 days of enrollment using KDIGO creatinine-based staging. SARS-CoV2 positive (CONFIRMED) were compared to SUSPECTED (negative or unknown). Outcome data was censored at 28-days.

RESULTS: In 331 patients of both sexes, 179 (54.1\%) were CONFIRMED, 4.2\% (14) died. AKI occurred in 124 (37.5\%) and severe AKI occurred in 63 (19.0\%). Incidence of AKI in CONFIRMED was 74/179 (41.3\%) versus 50/152 (32.9\%) for SUSPECTED; severe AKI occurred in $35(19.6 \%)$ of CONFIRMED and $28(18.4 \%)$ of SUSPECTED. Mortality was $6.2 \%(n=11)$ in CONFIRMED, but $9.5 \%(n=7)$ in those CONFIRMED with AKI. On multivariable analysis, only Hispanic ethnicity (relative risk $0.5,95 \% \mathrm{CI} 0.3-0.9$ ) was associated with less AKI development among those CONFIRMED.

CONCLUSIONS: AKI and severe AKI occur commonly in critically ill children with SARS-CoV2 infection, more than double the historical standard. Further investigation is needed during this continuing pandemic to describe and refine the understanding of pediatric AKI epidemiology and outcomes.

TRIAL REGISTRATION: NCT01987921.

Pediatric Research (2022) 91:1787-1796; https://doi.org/10.1038/s41390-021-01667-4

\section{IMPACT:}

What is the key message of the article?

- AKI occurs in children exposed to the novel SARS-CoV2 virus at high prevalence ( 40\% with some form of AKI and $20 \%$ with severe AKI).

\section{What does it add to the existing literature?}

- Acute kidney injury (AKI) occurs commonly in adult patients with SARS-CoV2 (COVID), very little data describes the epidemiology of AKI in children exposed to the virus.

\section{What is the impact?}

- A pediatric vaccine is not available; thus, the pandemic is not over for children. Pediatricians will need to manage significant end-organ ramifications of the novel SARS-CoV2 virus including AKI.

\section{INTRODUCTION}

Severe acute respiratory syndrome coronavirus 2 (SARS-CoV2) is associated with multiple end-organ effects in critically ill patients. Available, though still sporadic, data indicate children suffer infection at high rates and with increased morbidity, just as in adults $^{1-6}$. Recent data indicate organ dysfunction in the setting of SARS-CoV2 can occur in all populations, including children ${ }^{7-10}$, however, the nature of this relationship (SARS-CoV2 and organ injury) continues to be delineated. A large proportion ( $50 \%)$ of SARS-CoV2 infected adults requiring admission to intensive care

\footnotetext{
${ }^{1}$ Division of Critical Care, Children's Healthcare of Atlanta, Atlanta, GA, USA. ${ }^{2}$ Division of Nephrology, Children's Hospital of Alabama, Birmingham, AL, USA. ${ }^{3}$ Division of Cardiology, University of Colorado Anschutz School of Medicine, Children's Hospital of Colorado, Boulder, CO, USA. ${ }^{4}$ Division of Nephrology, Department of Pediatrics, Indiana University School of Medicine, Riley Hospital for Children, Indianapolis, IN, USA. ${ }^{5}$ Division of Pediatric Critical Care, Beaumont Children's Hospital, Royal Oak, MI, USA. ${ }^{6}$ Division of Nephrology, Department of Pediatrics, McMaster Children's Hospital, McMaster University, Hamilton, ON, Canada. ${ }^{7}$ Center for Acute Care Nephrology, Cincinnati Children's Hospital Medical Center, Cincinnati, OH, USA. ${ }^{8}$ Division of Nephrology, Hospital for Sick Children, Toronto, ON, Canada. A full list of SPARC Investigators members appears in the Supplementary Information. ${ }^{{ }}$email: Rajit.basu@choa.org
} 
units (ICU) develop acute kidney injury (AKI), with nearly $20 \%$ receiving renal replacement therapy (RRT). Those with AKI have increased morbidity versus similar patients without $\mathrm{AKI}^{11-17}$ and mortality is 2- to 9-fold higher. Delineation and detail of organ dysfunction in critically ill pediatric patients with SARS-CoV2 remain limited by the population size studied. Thus far, only preliminary data have been published describing AKI in the context of SARS-CoV2 ${ }^{3,18-22}$. Data from China indicate a total of 13 of $2133(0.6 \%)$ children in the Hubei province required ICU admission, though no data related to AKI is mentioned ${ }^{23}$. A convenience sample of nearly 50 children admitted to ICUs in the United States indicates $86 \%$ of children experienced at least one organ failure but AKI was not explicitly mentioned ${ }^{5}$.

We conducted a multinational, prospective, point-prevalence study in children and young adults admitted to pediatric ICU with suspected or confirmed SARS-CoV2 infection to study its implications with AKI. The aim of the study was to determine the difference in AKI prevalence in critically ill children between those with confirmed versus suspected SARS-CoV2 infection. The hypothesis, as in adults, was that in confirmed SARS-CoV2 infection would be associated with more and worse severity AKI and, consequently, more downstream morbidity and mortality compared to patients with suspected (i.e., unconfirmed) infection. In our initial, preliminary analysis we identified potential associations with chronic medical conditions and race with AKI in critically ill children suspected of infection with SARS-CoV2 $2^{22}-$ thus a secondary aim of this full study was to expand and strengthen or refute these findings.

\section{MATERIALS AND METHODS \\ Study design}

Multinational, prospective, point-prevalence study conducted from April 15, 2020 through June 24, 2020 (NCT:04466306, SARS-CoV2 Pediatric AKI Registry, and Collaborative-SPARC). Patient data were collected weekly from 57 participating centers and entered directly into a cloud-based data entry platform. Study data were collected and managed using REDCap (Research Electronic Data Capture) $^{24,25}$. The study was approved at each center by local Investigational Review Boards (with a requirement for patient consent waived) and data use agreements for data transfer were in place.

\section{Study population}

Overall, 57 participating centers throughout North America, South America, Europe, the Middle East, and Asia participated in SPARC-1 (Supplemental Table 1). All critically ill children $>1$ week and $\leq 25$ years of age admitted to the pediatric ICU receiving care in non-neonatal pediatric intensive care units (ICUs) (medical-surgical pediatric and cardiac) on the days of data capture were screened. Inclusion criteria were the following for patients in the ICU on the day of intake: (1) tested for SARS-CoV2 with symptomatology specific to respiratory disease or infectious appearance, (2) "enhanced droplet" precautions in place due to symptomatology (per institutional policy), (3) infectious disease service involvement for SARSCoV2 evaluation (per institutional policy), or (4) unexplained symptomatology concerning for SARS-CoV2 without having been tested yet (fever, shortness of breath, chest tightness, altered neurologic status). These determinations were made by the care provider in the local ICU and not subject to adjudication by the SPARC investigators (local or lead). There were no other exclusion criteria.

At 28-days after enrollment, diagnosis of SARS-CoV2 infection was confirmed on chart review for confirmation of polymerase chain reaction $(\mathrm{PCR}+)$ or antibody testing $(\mathrm{Ab}+)$ results based on local protocols. The tests used for detection changed through the course of the study (12 weeks) at each center as the pandemic evolved. A majority of the centers used multiple tests for the purpose of both PCR and Ab testingthese tests had evolving and improved sensitivity and specificity for detection of acute (PCR) or historical (Ab+) infection. Patients positive for either test were listed as CONFIRMED, while negative or unknown were listed as SUSPECTED (given the high proportion of false-negative rates related to the quality of testing available during the time of this study) ${ }^{26}$.

\section{Outcomes}

The primary outcome of interest was AKI prevalence defined by the weekly rate of AKI in SUSPECTED or CONFIRMED children for that given week and then overall for the entirety of the study. Kidney Disease Improving Global Outcomes (KDIGO) criteria based on serum creatinine rise relative to baseline creatinine was used to denote AKI. Weekly serum creatinine values were obtained while patients were in the study. Baseline creatinine was defined as the lowest serum creatinine within the three months prior to hospital admission. If baseline creatinine was not available, the validated height-independent equations for age and sex were used to back-calculate creatinine, assuming normal pre-admission estimated glomerular filtration rate (eGFR) $\left(120 \mathrm{ml} / \mathrm{min} / 1.73 \mathrm{~m}^{2}\right.$ for children $\geq 2$ years of age; median normative-based eGFR-for-age in children $<2$ years old) ${ }^{27}$. Secondary outcomes included AKI severity (Stage based by KDIGO scoring), critical care resources used: vasoactive, invasive ventilator support, renal replacement therapy (RRT), extracorporeal membrane oxygenation (ECMO), length of hospitalization (dichotomized at less than or greater than 14 days), and 28-days hospital mortality.

\section{Covariates}

Patient demographic data, center-level data, and additional clinical factors obtained on individual patients included comorbidities, baseline serum creatinine and eGFR, admission serum creatinine, and categories of reasons for ICU admission (shock/hemodynamic instability, sepsis/infection, respiratory distress, neurologic symptoms, gastrointestinal symptoms, other).

\section{Statistical analyses}

The prevalence of AKI amongst SUSPECTED or CONFIRMED SARS-CoV2 infection in critically ill children was analyzed using descriptive statistics. Comparisons were made with Chi-square, Fisher's exact test, and unpaired $t$-tests as appropriate.

Among those CONFIRMED with SARS-CoV2 infection, further exploration was conducted to evaluate for independent risk factors of AKI development. We used chi-square, and Fisher's Exact test as appropriate, to evaluate for differences between those with and without AKI. Univariate risk differences and risk ratios with corresponding 95\% confidence intervals were calculated. We present both risk differences and risk ratios for univariate analysis, but only risk ratios for multivariable analysis. A logbinomial regression model was used for multivariable analysis among those CONFIRMED. For multivariate analyses, we included covariates with a $p$-value of $\leq 0.2$ in univariate analyses, and a priori forced the following potential confounders into the model (center, age categories, sex, and presence or absence of comorbidities). Continuous variables were also condensed to larger categorical groupings to allow model stability. Alphalevel of $<0.05$ was considered statistically significant. A sensitivity analysis was conducted for the multivariable model among the entire cohort (SUSPECTED and CONFIRMED). All statistical analyses were performed in SAS, version 9.4 (SAS Institute, Inc., Cary, North Carolina).

\section{RESULTS}

\section{Characteristics of cohort}

A total of 331 patients were included from 42 of the participating centers in 15 countries (Table 1). In these patients, 179/331 (54.1\%) had CONFIRMED SARS-CoV2 infection. Over 70\% $(n=233)$ had at least one chronic condition (108 (71.1\%) SUSPECTED versus 125 $(69.8 \%)$ CONFIRMED). The most common chronic conditions included central nervous system dysfunction and pulmonary disease. Sex, age, and body mass index were not different between those with CONFIRMED infection versus SUSPECTED infection. However, those of racial/ethnic minorities were more likely to have CONFIRMED infection (36.9\% among Hispanics, $27.9 \%$ among Blacks) versus SUSPECTED infection (12.5\% among Hispanics, 22.5\% among Blacks), $p$-values $<0.0001$ and 0.04, respectively.

\section{Prevalence and risk factors for acute kidney injury in the context of SARS-CoV2 infection}

AKI was common in the patients enrolled ( $n=124,37.4 \%)$ (Table 2). No statistically significant differences were found in 
Table 1. Epidemiology of SARS-CoV2 infection in critically ill children and young adults.

\begin{tabular}{|c|c|c|c|}
\hline Characteristics & Total & Suspected & Confirmed \\
\hline$N$ & 331 & 152 & 179 \\
\hline \multicolumn{4}{|l|}{ Date of enrollment } \\
\hline Week 1 (April 15) & $47(14.2)$ & $13(8.6)$ & $34(19.0)$ \\
\hline Week 2 (April 22) & $39(11.8)$ & $23(15.1)$ & $16(8.9)$ \\
\hline Week 3 (April 29) & $29(8.8)$ & $17(11.2)$ & $12(6.7)$ \\
\hline Week 4 (May 6) & $29(8.8)$ & $13(8.6)$ & $16(8.9)$ \\
\hline Week 5 (May 13) & $43(13.0)$ & $20(13.2)$ & $23(12.9)$ \\
\hline Week 6 (May 20) & $33(10.0)$ & $13(8.6)$ & $20(11.2)$ \\
\hline Week 7 (May 27) & $21(6.3)$ & $11(7.2)$ & $10(5.6)$ \\
\hline Week 8 (June 3) & $23(7.0)$ & $15(9.9)$ & $8(4.5)$ \\
\hline Week 9 (June 10) & $23(7.0)$ & $10(6.6)$ & $13(7.3)$ \\
\hline Week 10 (June 17) & $17(5.1)$ & $7(4.6)$ & $10(5.6)$ \\
\hline Week 11 (June 24) & $27(8.2)$ & $10(6.6)$ & $17(9.5)$ \\
\hline $\begin{array}{l}\text { Age, years } \\
\text { (median, IQR) }\end{array}$ & $11(3,16)$ & $10(2,16)$ & $12(4,16)$ \\
\hline \multicolumn{4}{|l|}{ Age categories } \\
\hline $\begin{array}{l}\text { Infants/young } \\
\text { children ( } 0- \\
<5 \text { years) }\end{array}$ & $100(30.2)$ & $52(34.2)$ & $48(26.8)$ \\
\hline $\begin{array}{l}\text { School-aged } \\
\text { children (5- } \\
<13 \text { years) }\end{array}$ & $87(26.3)$ & $38(25.0)$ & $49(27.4)$ \\
\hline $\begin{array}{l}\text { Adolescents/ } \\
\text { young adults } \\
\text { (13-25 years) }\end{array}$ & $144(43.5)$ & $62(40.8)$ & $82(45.8)$ \\
\hline \multicolumn{4}{|l|}{ Sex } \\
\hline Female & $146(44.1)$ & $64(42.1)$ & $82(45.8)$ \\
\hline Male & $185(55.9)$ & $88(57.9)$ & $97(54.2)$ \\
\hline \multicolumn{4}{|l|}{ Race } \\
\hline Caucasian & $159(48.0)$ & $86(56.6)$ & $73(40.8)$ \\
\hline Black & $84(25.4)$ & $34(22.4)$ & $50(27.9)$ \\
\hline Other & $9(2.7)$ & $3(2.0)$ & $6(3.4)$ \\
\hline Unknown & 79 (23.9) & $29(19.1)$ & $50(27.9)$ \\
\hline \multicolumn{4}{|l|}{ Ethnicity } \\
\hline $\begin{array}{l}\text { Non-Hispanic, } \\
\text { non-Latino, non- } \\
\text { Spanish }\end{array}$ & $195(58.9)$ & $108(71.1)$ & 87 (48.6) \\
\hline $\begin{array}{l}\text { Hispanic, Latino, } \\
\text { Spanish }\end{array}$ & $85(25.7)$ & $19(12.5)$ & $66(36.9)$ \\
\hline Unknown & $51(15.4)$ & $25(16.5)$ & $26(14.5)$ \\
\hline \multicolumn{4}{|c|}{ Body mass index categories ${ }^{a}$} \\
\hline Underweight & $36(10.9)$ & $16(10.5)$ & $20(11.2)$ \\
\hline Normal weight & $118(35.7)$ & $57(37.5)$ & $61(34.1)$ \\
\hline Overweight & $30(9.1)$ & $9(5.9)$ & $21(11.7)$ \\
\hline Obese & 79 (23.9) & $29(19.1)$ & $50(27.9)$ \\
\hline \multicolumn{4}{|l|}{ Location } \\
\hline United States & $298(90.0)$ & $125(82.2)$ & $173(96.7)$ \\
\hline Canada & $13(3.9)$ & $13(8.6)$ & $0(0)$ \\
\hline Western Europe & $9(2.7)$ & $7(4.6)$ & $2(1.1)$ \\
\hline $\begin{array}{l}\text { Eastern Europe/ } \\
\text { Russia }\end{array}$ & $10(3.0)$ & $6(4.0)$ & $4(2.2)$ \\
\hline Middle East & $<5$ & $\mathrm{n} / \mathrm{a}$ & $\mathrm{n} / \mathrm{a}$ \\
\hline $\begin{array}{l}\text { Any chronic } \\
\text { condition }^{\text {b }}\end{array}$ & $233(70.4)$ & $108(71.1)$ & $125(69.8)$ \\
\hline
\end{tabular}

\section{Table 1 continued}

\begin{tabular}{|c|c|c|c|}
\hline Characteristics & Total & Suspected & Confirmed \\
\hline $\begin{array}{l}\text { No chronic } \\
\text { condition }\end{array}$ & $98(29.6)$ & $44(29.0)$ & $54(30.2)$ \\
\hline Asthma & 42 (12.7) & 19 (12.5) & $23(12.8)$ \\
\hline Seizures/epilepsy & $44(13.3)$ & $22(14.5)$ & $22(12.3)$ \\
\hline $\begin{array}{l}\text { Congenital heart } \\
\text { disease (corrected } \\
\text { and uncorrected) }\end{array}$ & $30(9.1)$ & $12(7.9)$ & $18(10.1)$ \\
\hline $\begin{array}{l}\text { Cancer (in therapy, } \\
\text { remission) }\end{array}$ & $29(8.8)$ & $12(7.9)$ & $17(9.5)$ \\
\hline $\begin{array}{l}\text { Cerebral palsy/ } \\
\text { encephalopathy }\end{array}$ & $34(10.3)$ & $18(11.8)$ & $16(8.9)$ \\
\hline Chronic ventilation & $36(10.9)$ & $21(13.8)$ & $15(8.4)$ \\
\hline Diabetes & $18(5.4)$ & $6(3.9)$ & $12(6.7)$ \\
\hline Hypertension & $13(3.9)$ & $8(5.3)$ & $5(2.8)$ \\
\hline $\begin{array}{l}\text { Baseline eGFR, } \\
\text { median (IQR) }^{\mathrm{c}}\end{array}$ & $\begin{array}{l}125 \\
(93,180)\end{array}$ & $\begin{array}{l}126 \\
(94,198)\end{array}$ & $\begin{array}{l}125 \\
(91,157)\end{array}$ \\
\hline $\begin{array}{l}\text { Baseline serum } \\
\text { creatinine, } \mathrm{mg} / \mathrm{dl} \text {, } \\
\text { median }(\mathrm{QQR})^{\mathrm{c}}\end{array}$ & $\begin{array}{l}0.38 \\
(0.23,0.66)\end{array}$ & $\begin{array}{l}0.32 \\
(0.22,0.66)\end{array}$ & $\begin{array}{l}0.47 \\
(0.23,0.66)\end{array}$ \\
\hline $\begin{array}{l}\text { Admission serum } \\
\text { creatinine, } \mathrm{mg} / \mathrm{dl} \text {, } \\
\text { median (range) }\end{array}$ & $\begin{array}{l}0.52 \\
(0.33,0.81)\end{array}$ & $\begin{array}{l}0.50 \\
(0.30,0.81)\end{array}$ & $\begin{array}{l}0.59 \\
(0.37,0.80)\end{array}$ \\
\hline \multicolumn{4}{|l|}{ Admission reasons } \\
\hline $\begin{array}{l}\text { Shock/ } \\
\text { hemodynamic } \\
\text { instability }\end{array}$ & $91(27.5)$ & $32(21.1)$ & $59(33.0)$ \\
\hline Sepsis/infection & $77(23.3)$ & $39(25.7)$ & $38(21.2)$ \\
\hline $\begin{array}{l}\text { Respiratory } \\
\text { distress }\end{array}$ & $159(48.0)$ & $69(45.4)$ & $90(50.3)$ \\
\hline CNS symptoms & 39 (11.8) & $23(15.1)$ & $16(8.9)$ \\
\hline $\begin{array}{l}\text { Metabolic } \\
\text { derangements }\end{array}$ & $18(5.4)$ & $7(4.6)$ & $11(6.2)$ \\
\hline $\begin{array}{l}\text { Gastrointestinal } \\
\text { symptoms }\end{array}$ & $10(3.0)$ & $3(2.0)$ & 7 (3.9) \\
\hline Other & $48(14.5)$ & $26(17.1)$ & $22(12.3)$ \\
\hline \multicolumn{4}{|l|}{ Acute kidney Injury } \\
\hline None & $207(62.5)$ & $102(67.1)$ & 105 (58.7) \\
\hline Mild (stage 1) & $61(18.4)$ & $22(14.5)$ & $39(21.8)$ \\
\hline Severe (stage $2 / 3$ ) & $63(19.0)$ & $28(18.4)$ & 35 (19.6) \\
\hline
\end{tabular}

Data presented as $N$ (column percentages) except where indicated. AKI acute kidney injury, eGFR estimated glomerular filtration rate $(\mathrm{ml} / \mathrm{min} /$ $\left.1.73 \mathrm{~m}^{2}\right), I Q R$ interquartile range.

${ }^{\mathrm{a}} \mathrm{BMI} /$ weight-for-height data missing for 68 patients. BMI categories based on CDC definitions; weight-for-height for those $<2$ years of age, BMI percentile for those $2-20$ years, and adult BMl categories for those $>20$ years.

${ }^{\mathrm{b}}$ Common chronic conditions presented for those where at least 10 patients had the condition.

'Baseline eGFR $\left(\mathrm{ml} / \mathrm{min} / 1.73 \mathrm{~m}^{2}\right) /$ creatinine $(\mathrm{mg} / \mathrm{dl})$ missing for 204 patients, so for the remainder, it was estimated based on standard estimating equations (see "Methods" section).

${ }^{d}$ Admission creatinine value missing on four patients.

sex, presence of chronic conditions, or body mass index between those with or without AKI for the entire cohort. Racial and ethnic minorities had a higher percentage with AKI than White patients ( $p$-value $=0.04$ for Blacks, 0.03 for Hispanics).

AKI occurred in 74/179 (41.3\%) CONFIRMED patients compared to $50 / 152$ (32.9\%) SUSPECTED patients (Table 2). Among those with SUSPECTED SARS-CoV2, AKI occurred more frequently in 
Table 2. AKI prevalence in critically ill children and young adults with suspected SARS-CoV2 infection.

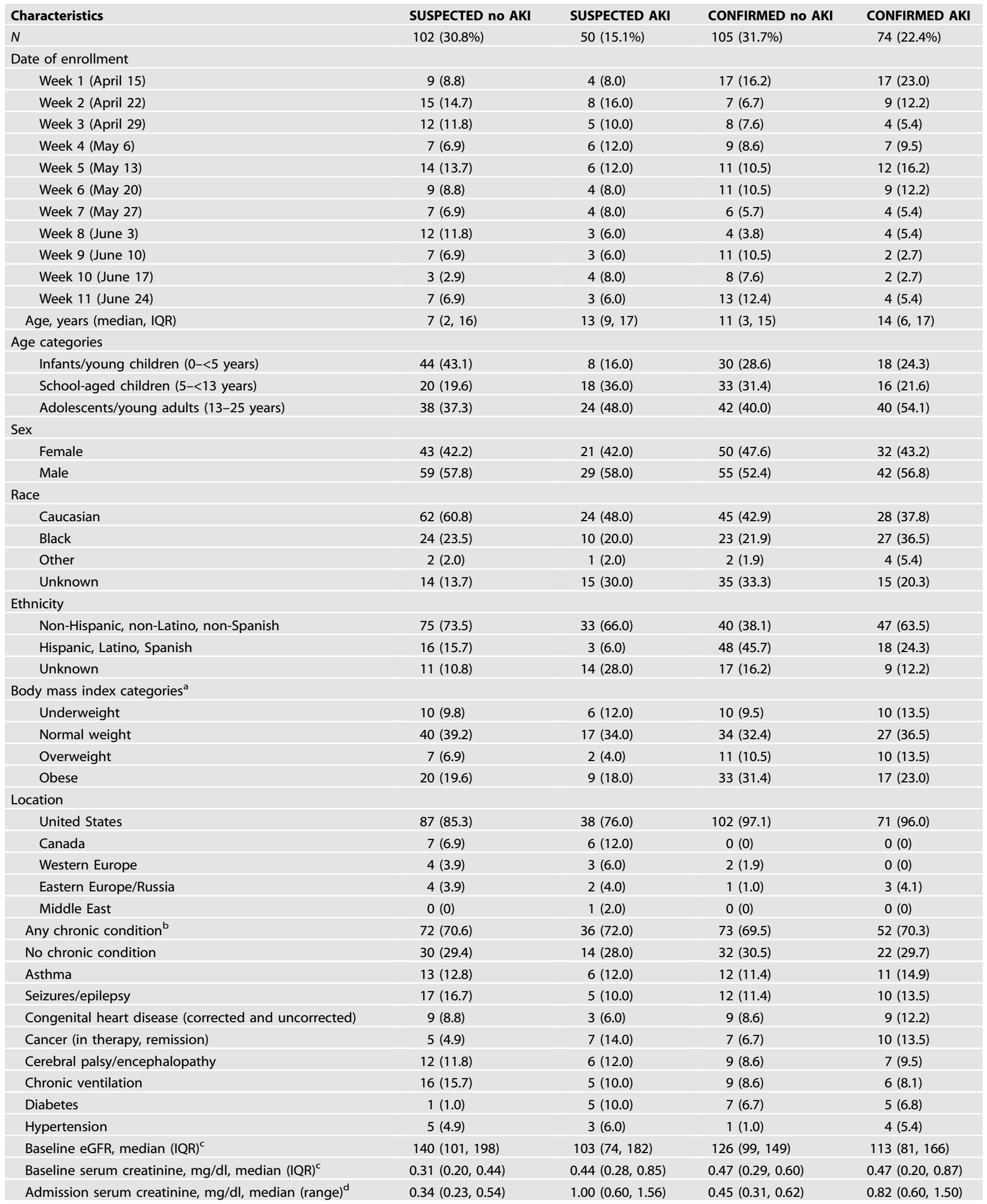


Table 2 continued

\begin{tabular}{|l|}
\hline Characteristics \\
\hline Admission reasons \\
Shock/hemodynamic instability \\
Sepsis/infection \\
\hline Respiratory distress \\
\hline CNS symptoms \\
\hline Metabolic derangements \\
Gastrointestinal symptoms \\
\hline Other \\
\hline
\end{tabular}

\section{SUSPECTED no AKI}

$14(13.7)$

19 (18.6)

$53(52.0)$

$21(20.6)$

$2(2.0)$

$1(1.0)$

$16(15.7)$
SUSPECTED AKI

$18(36.0)$
$20(40.0)$
$16(32.0)$
$2(4.0)$
$5(10.0)$
$2(4.0)$
$10(20.0)$

CONFIRMED no AKI

CONFIRMED AKI

Data presented as $N$ (column percentages) except where indicated.

$A K I$ acute kidney injury, eGFR estimated glomerular filtration rate $\left(\mathrm{ml} / \mathrm{min} / 1.73 \mathrm{~m}^{2}\right), I Q R$ interquartile range.

${ }^{\mathrm{a}} \mathrm{BMI} /$ weight-for-height data missing for 68 patients. BMI categories based on CDC definitions; weight-for-height for those $<2$ years of age, BMI percentile for those $2-20$ years, and adult BMI categories for those $>20$ years.

${ }^{\mathrm{b}} \mathrm{Common}$ chronic conditions presented for those where at least 10 patients had the condition.

'Baseline eGFR $\left(\mathrm{ml} / \mathrm{min} / 1.73 \mathrm{~m}^{2}\right) / \mathrm{creatinine}(\mathrm{mg} / \mathrm{dl})$ missing for 204 patients, so for the remainder, it was estimated based on standard estimating equations (see "Methods" section).

${ }^{\mathrm{d} A d m i s s i o n}$ creatinine value missing on four patients.

older children compared to younger children ( $p$-value $<0.001)$, but this association was not found among those with CONFIRMED SARS-CoV2 ( $p$-value $=0.08$ ). Hispanic ethnicity was found to be protective against AKI among CONFIRMED patients ( $p$-value $<$ 0.001 ), but not among SUSPECTED patients ( $p$-value $=0.27)$. AKI was associated with shock to a greater percentage than without shock among both cohorts ( $p$-value $<0.01$ among SUSPECTED, $p$-value $<0.001$ among CONFIRMED). However, other admission diagnoses associated with increased risk of AKI were only significant among the SUSPECTED cohort and not the CONFIRMED cohort (sepsis/infection, respiratory distress, central nervous system symptoms, and metabolic derangements). In univariate analyses, Hispanic ethnicity was protective ( $p$-value $<0.001)$, while invasive respiratory support and vasopressor use were associated with an increased risk of AKI development ( $p$-value 0.02 and $<0.01$, respectively) (Table 2). The use of vasopressors was associated with a $23.8 \%$ absolute increase in the risk of developing AKI compared to no vasopressor use (risk difference $23.8 \%, 95 \% \mathrm{Cl}$ 6.9-40.6\%).

After adjustment for covariates, only the presence of Hispanic ethnicity was associated with AKI in CONFIRMED patients (protective), adjusted RR 0.5 (95\% Cl 0.3-0.9, Table 3). Vasopressor use was not associated with an increased risk of AKI development $(p$-value $=0.92)$.

Additional analyses were done to evaluate adjusted risk ratios for AKI development among the entire cohort (CONFIRMED and SUSPECTED) (Table 4). Adjusting for the same variables as in the confirmed cohort, we found that Hispanic ethnicity was no longer a protective factor $(p$-value $=0.19)$, yet the presence of CNSrelated admission diagnosis was protective ( $p$-value $=0.03)$. This suggests that there is effect modification of SARS-CoV2 confirmation and the risk factors associated with the development of AKI.

\section{Severity of acute kidney injury and outcomes in the context of SARS-CoV2 infection}

Injury severity varied amongst those with AKI, with $18.4 \%$ having mild or stage $1 \mathrm{AKI}$ and $19.0 \%$ having severe (stage 2 or 3 ) AKI (Table 5). Among the entire cohort, children with severe AKI or mild AKI were older compared to those without AKI ( $p$-value 0.01 and 0.002 , respectively). In the CONFIRMED population, 74 had any severity of AKI in the first 14 days (41.4\%).

AKI in the setting of SUSPECTED and CONFIRMED SARS-CoV2 infection, was associated with increased morbidity (Fig. 1). Among CONFIRMED patients, a greater percentage of those with AKI required respiratory and vasoactive support compared to those without AKI, $35.1 \%$ versus $20.0 \%$ ( $p$-value $<0.05$ ) and $33.8 \%$ versus
Table 3. Independent risk factors for AKI in critically ill children and young adults with suspected SARS-CoV2 infection.

\begin{tabular}{|lll|}
\hline $\begin{array}{l}\text { Age category } \\
<5 \text { years }\end{array}$ & Relative risks & -value \\
\hline $5-13$ years & Reference & \\
\hline 13 years & $1.1(0.8-1.6)$ & 0.5 \\
\hline $\begin{array}{l}\text { Gender } \\
\text { Male }\end{array}$ & $0.6(0.4-1.1)$ & 0.09 \\
\hline Female & $1.2(0.9-1.6)$ & 0.3 \\
\hline Race & Reference & \\
\hline Black & & \\
\hline White & $1.5(0.7-3.0)$ & 0.3 \\
\hline Ethnicity & Reference & \\
\hline Hispanic & & \\
\hline Not-Hispanic & $0.7(0.4-1.2)$ & 0.19 \\
\hline Presence of Comorbidities & Reference & \\
\hline Healthy & $0.9(0.6-1.3)$ & 0.6 \\
\hline Admission diagnosis & Reference & \\
\hline CNS-related & & \\
\hline Not CNS-related & $0.4(0.1-0.9)$ & 0.03 \\
\hline Vasopressor use & Reference & \\
\hline No vasopressor use & $1.4(0.9-2.0)$ & 0.10 \\
\hline Invasive respiratory Support & Reference \\
\hline No invasive respiratory support & $1.3(0.9-1.9)$ & 0.11 \\
\hline
\end{tabular}

The above data demonstrate the relative risks versus embedded reference value for the association of SARS-CoV2 infection with the outcome of AKI. Variables in the model included center, age category, gender, race, ethnicity, presence or absence of comorbidities, CNS (central nervous system)-associated admission diagnosis, invasive respiratory use, and vasopressor use.

$16.2 \%$ ( $p$-value $<0.01)$, respectively. While among those with SUSPECTED SARS-CoV2 infection, only vasoactive support occurred more frequently in those with AKI compared to those without AKI $(44.0 \%$ versus $13.7 \%, p$-value $<0.001)$. The majority of children in the study had hospital length of stay (LOS) under 14 days, regardless of AKI status. The confirmation of SARS-CoV2 infection or AKI did not confer a higher proportion of patients with a LOS $>14$ days. Mortality was $4.2 \%(n=14)$ in the overall study population. Though this trend increased to $6.2 \%(n=11)$ for patients with CONFIRMED infection, and further to $9.5 \%(n=7)$ 
Table 4. Estimates of effect size for AKI development among critically ill children and young adults with confirmed SARS-CoV2 infection.

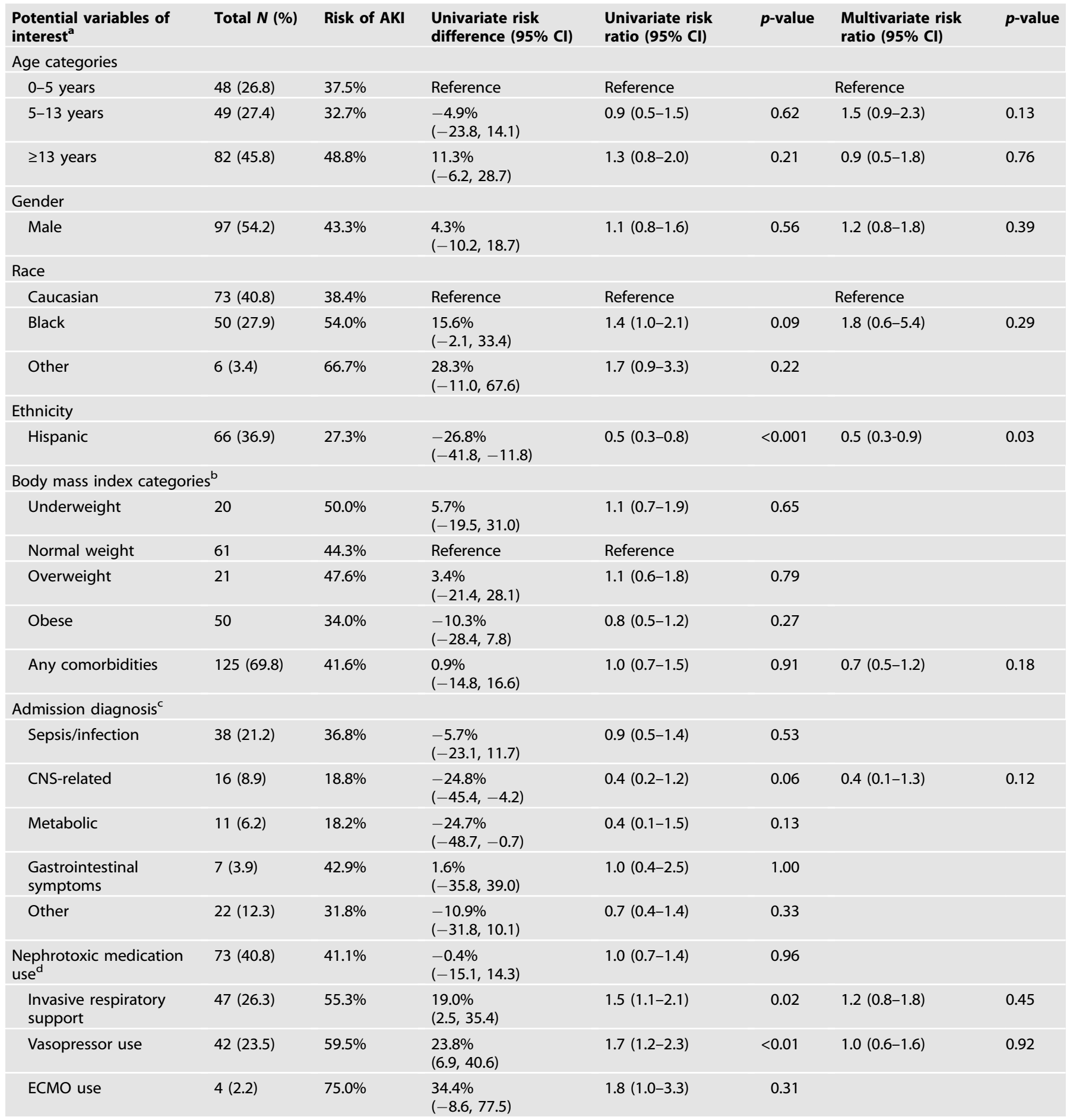

Table presents the univariate risk differences and risk ratios with corresponding $95 \%$ confidence intervals. The multivariate model includes age category, gender, center, race, ethnicity, presence or absence of comorbidities, CNS-associated admission diagnosis, invasive respiratory use, and vasopressor use. $A K I$ acute kidney injury, $\mathrm{Cl}$ confidence interval, CNS central nervous system, ECMO extracorporeal membrane oxygenation.

${ }^{a}$ For variables where a reference category is not specified in the table, the references are as follows: Gender reference is female. The ethnicity reference is nonHispanic. Comorbidities reference is no comorbidities. Reference for admission diagnoses is the absence of that diagnosis. Reference for nephrotoxic medication use is the absence of use, and similarly for invasive respiratory support, vasopressor use, and ECMO use.

${ }^{\mathrm{b}}$ Body mass index categories missing for 27 patients.

${ }^{\mathrm{C}}$ Admission diagnosis categories were not mutually exclusive and patients could have multiple diagnoses.

${ }^{\mathrm{d}}$ Nephrotoxic medications were defined based on the nephrotoxic injury negated by the just-in-time action (NINJA) initiative list. 
Table 5. Severity of AKI in critically ill children and young adults with suspected SARS-CoV2 infection.

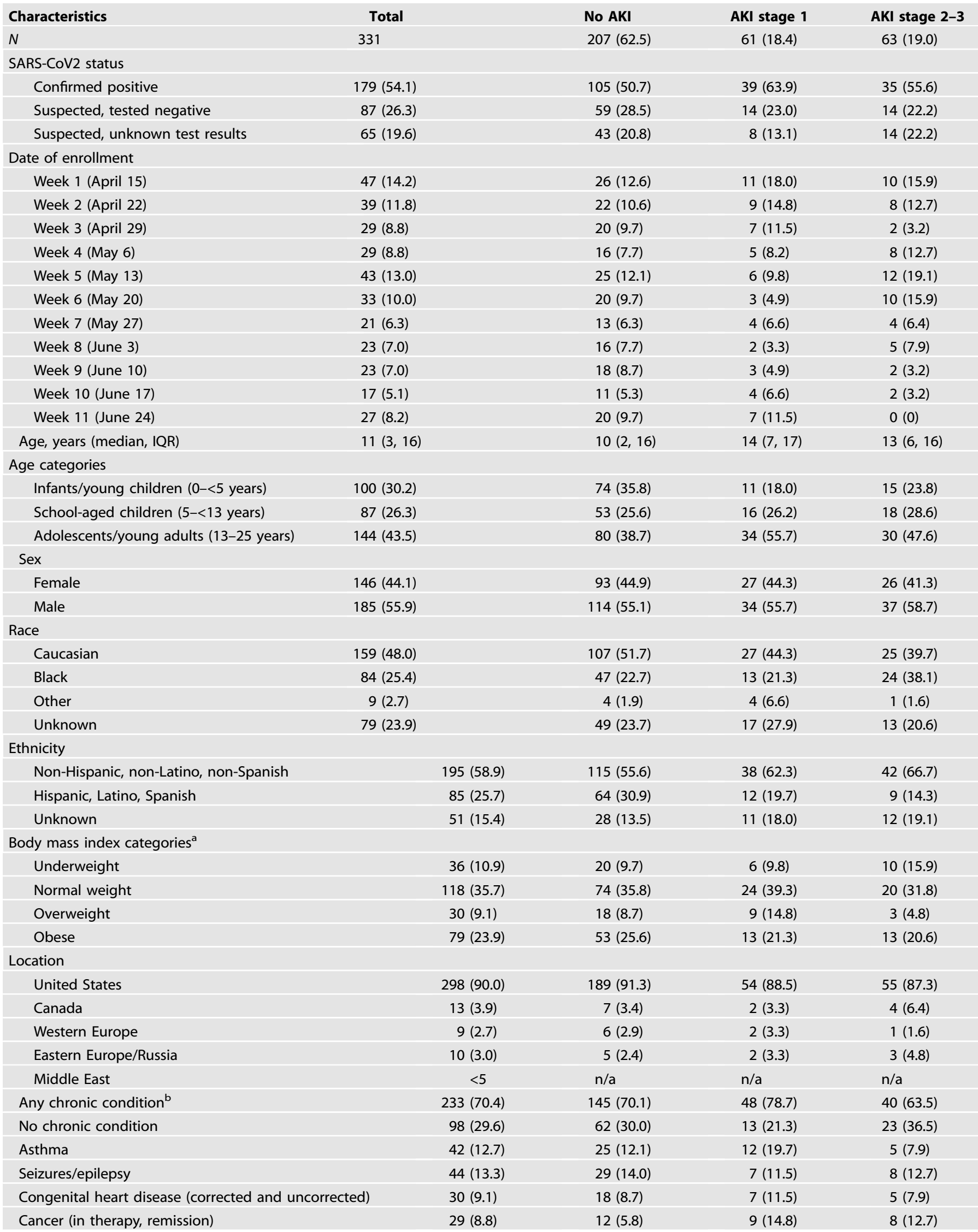


Table 5 continued

Characteristics
Cerebral palsy/encephalopathy
Chronic ventilation
Diabetes
Hypertension
Baseline eGFR, median (IQR)
Baseline serum creatinine, $\mathrm{mg} / \mathrm{dl}$, median $(\mathrm{IQR})^{\mathrm{c}}$
Admission serum creatinine, $\mathrm{mg} / \mathrm{dl}$, median $(\mathrm{range})^{\mathrm{d}}$
Admission reasons
Shock/hemodynamic instability
Sepsis/infection
Respiratory distress
CNS symptoms
Metabolic derangements
Gastrointestinal symptoms
Other

\section{Total}

$34(10.3)$
$36(10.9)$
$18(5.4)$
$13(3.9)$
$125(93,180)$
$0.38(0.23,0.66)$
$0.52(0.33,0.81)$

\section{No AKI}

$21(10.1)$

$25(12.1)$

$8(3.9)$

6 (2.9)

$129(100,181)$

$0.34(0.22,0.58)$

$0.40(0.26,0.60)$

AKI stage 1
$5(8.2)$
$7(11.5)$
$5(8.2)$
$3(4.9)$
$112(77,159)$
$0.39(0.27,1.11)$
$0.76(0.50,1.00)$

AKI stage 2-3

$8(12.7)$

$4(6.3)$

5 (7.9)

$4(6.3)$

$108(79,196)$

$0.50(0.20,0.72)$

$1.27(0.74,2.37)$

\begin{tabular}{|cc|}
\hline $91(27.5)$ & $38(18.4)$ \\
$77(23.3)$ & $43(20.8)$ \\
$159(48.0)$ & $103(49.8)$ \\
\hline $39(11.8)$ & $34(16.4)$ \\
$18(5.4)$ & $11(5.3)$ \\
$10(3.0)$ & $5(2.4)$ \\
$48(14.5)$ & $31(15.0)$
\end{tabular}

$32(50.8)$

$23(36.5)$

26 (41.3)

3 (4.8)

2 (3.2)

3 (4.8)

9 (14.3)

Data presented as $N$ (column percentages) except where indicated.

$A K I$ acute kidney injury, eGFR estimated glomerular filtration rate $\left(\mathrm{ml} / \mathrm{min} / 1.73 \mathrm{~m}^{2}\right), I Q R$ interquartile range.

${ }^{\mathrm{a}} \mathrm{BMI} /$ weight-for-height data missing for 68 patients. BMI categories based on CDC definitions; weight-for-height for those $<2$ years of age, BMI percentile for those 2-20 years, and adult BMI categories for those $>20$ years.

${ }^{\mathrm{b}} \mathrm{Common}$ chronic conditions presented for those where at least 10 patients had the condition.

'Baseline eGFR (ml/min/1.73 $\mathrm{m}^{2}$ ) / creatinine ( $\left.\mathrm{mg} / \mathrm{dl}\right)$ missing for 204 patients, so for the remainder it was estimated based on standard estimating equations (see "Methods" section).

${ }^{\mathrm{d}}$ Admission creatinine value missing on four patients.

among those CONFIRMED and AKI, these did not reach statistical significance between groups $(p=0.09$ in CONFIRMED versus SUSPECTED and $p=0.049$ in CONFIRMED versus ALL).

Clinical management details do not seem to suggest differences between the groups. Nephrotoxin medication use was slightly higher in those with AKI among SUSPECTED SARS-CoV2 infection but it was not statistically significant $(60.0 \%$ versus $44.1 \%, p$-value $=0.06$ ) and there was no difference in nephrotoxic medication use between those with and without AKI among the CONFIRMED cohort $(40.5 \%$ versus $41.0 \%, p$-value $=0.96)$. Remdisivir was only used in 9 patients in the entire cohort, all with confirmed SARS-CoV2 infection (5.0\%), and only 3 of those with AKI (4.1\%). Maximum fluid overload percentage was similar between those with and without AKI in CONFIRMED cohort (median 2.8\%, IQR 0.1-8.4\%; median 2.9\%, IQR 0.3-9.1\%, respectively) and the SUSPECTED cohort (median 1.1\%, IQR 0-5.4\%; median 20\%, IQR 0.3-4.1\%).

\section{DISCUSSION}

$\mathrm{AKI}$ is common in pediatric and young adult patients in the setting of potential infection with the SARS-CoV2 virus. The AKI we describe in children tested for SARS-CoV2 infection appears to be higher in incidence versus historical norms-both in terms of overall percentage and severity. Compared to the global epidemiology study of AKI in 4914 critically ill children $\left(\right.$ AWARE $^{28}$ ), the overall AKI incidence in SPARC was $41.4 \%$ (versus $26.2 \%$ in AWARE) and was $19 \%$ for severe AKI (versus $11.6 \%$ in AWARE). We found that children with CONFIRMED infection had a higher, but not statistically significant, prevalence of AKI than those with SUSPECTED infection. In a sepsis sub-cohort analysis of the AWARE data set $(n=765)$, AKI occurred in $40.5 \%$ of children and $22.3 \%$ was severe $\mathrm{AKI}^{29}$. Mortality in this analysis was $4.2 \%$ overall $(6.2 \%$ in CONFIRMED children, $9.5 \%$ in those with CONFIRMED and AKI) compared to historical numbers (AWARE: $2.5 \%$ in ICU patients and $11.0 \%$ patients with AKI). A similar association between sepsisassociated AKI and mortality was reported in AWARE (7.7\%) versus the current study with CONFIRMED and AKI. In a point-prevalence study of pediatric sepsis, $25 \%$ of 569 patients captured had $\mathrm{AKI}^{30,31}$. Additionally, we identified a cohort of children requiring RRT, as in adult AKI associated with SARS-CoV2.

We were able to further refine risk factors for AKI in the context of SARS-CoV2 infection. We found a similar AKI occurrence rate in our patients with confirmed SARS-CoV2 infection $(41.4 \%$ for any $\mathrm{AKI}$ and $19.6 \%$ for severe $\mathrm{AKI})$. In the available literature related to AKI in the context of SARS-CoV2, we note that our data elaborates on the preliminary findings that we had reported earlier in this cohort ${ }^{22}$. Our study expands on our previous data and provides more granularity to findings from smaller pediatric studies, which showed variability in AKI rates (from 0 to $40 \% \mathrm{AKI}$ rate in cohorts $<75$ total patients studied ${ }^{5,32,33}$ ). It remains unclear at this time how the AKI epidemiology in children affected by the novel virus has changed during subsequent waves as there remains very little published data. Finally, distinct from our interim analysis, we found that Hispanic ethnicity and primary diagnosis of central nervous system dysfunction was associated with less AKI, different than our preliminary analysis, which was limited by population size for the purpose of adjusting associations for multivariate analysis. The rationale for the protection is unclear as it is not completely congruous with data relating risks to pediatric AKI. Hispanic race in AWARE showed no association with increased or decreased protection from AKI. A protective association may have been delineated in this sample of patients based on an increased proportion of Hispanic patients testing positive for SARS-CoV2 on incidental testing (versus symptomatic). Central nervous system dysfunction as a primary diagnosis also may actually be associated with "lower" AKI prevalence. Many patients with altered mental status, seizures, or drug ingestions changing CNS status are at lower risk for $\mathrm{AKI}^{34}$. Emerging data indicate SARS-CoV2 is associated with an increased risk of new or additive CNS dysfunction, but this is potentially outside the scope of $\mathrm{AKI}^{35}$.

The role of chronic disease and SARS-CoV2 has been controversial. In our preliminary analysis of this data, we had indicated an early connection between chronic respiratory and 


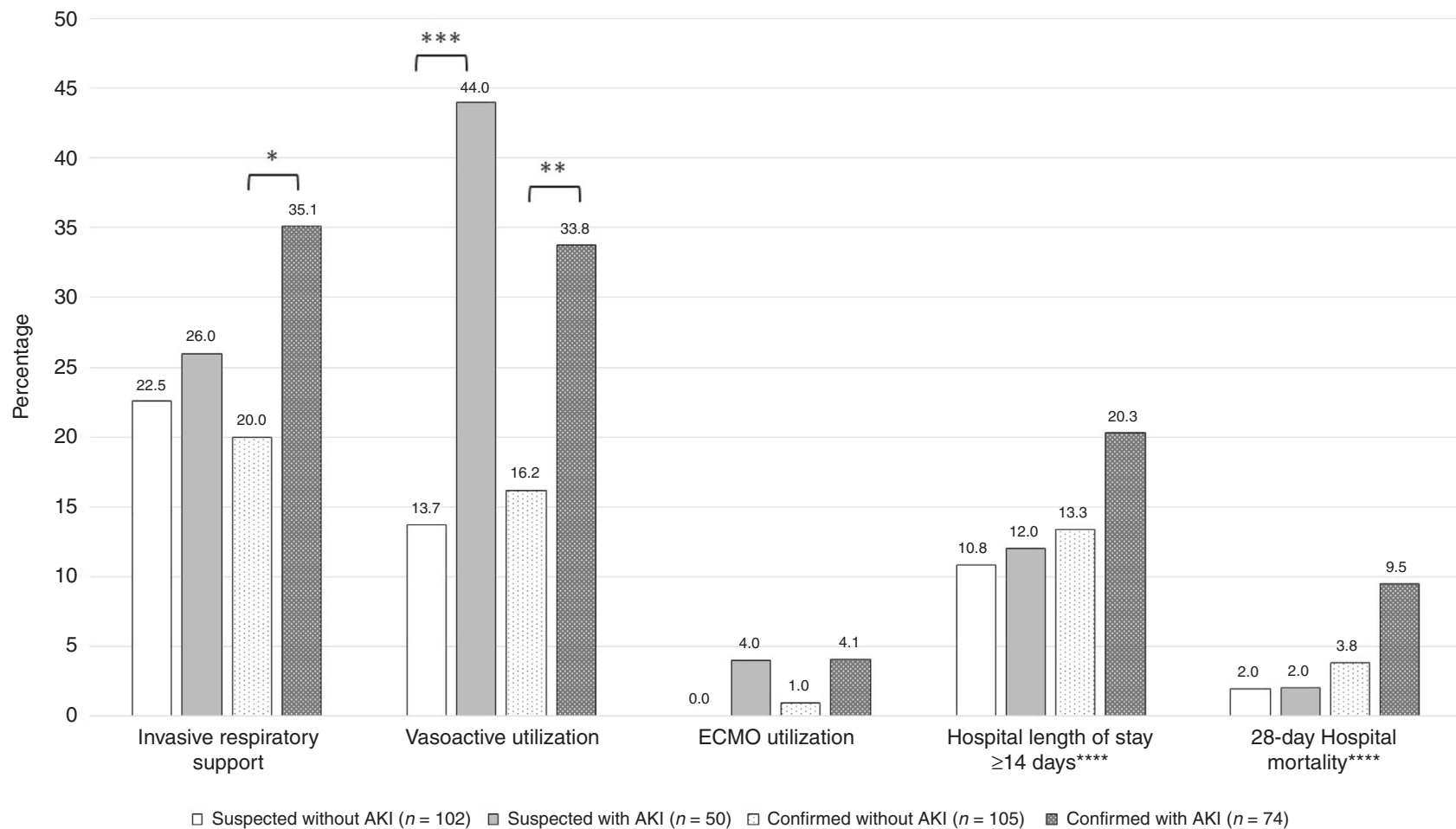

Fig. 1 Critical care outcomes for critically ill children and young adults with confirmed and suspected SARS-CoV2 infection and by AKI status. Percentage of patients in the given category presented on $Y$ axis. $X$ axis presents various clinical outcomes by cohort; suspected of SARS-CoV2 without AKI, $n=331$; suspected of SARS-CoV2 with AKI; confirmed with SARS-CoV2 without AKI, $n=$ confirmed with SARS-CoV2 with AKI, $n=74$. ${ }^{*} p$-value $<0.05$. ${ }^{* *} p$-value $<0.01$. ${ }^{* * *} p$-value $<0.001$. ${ }^{* * *}$ Length of hospitalization among survivors, but missing for 46 survivors and hospital mortality outcome missing for 34 patients. AKI acute kidney injury, ECMO extracorporeal membrane oxygenation.

neurologic disease and $\mathrm{AKI}$ in the setting of viral infection, but we did not show such an association in our expanded findings. Interestingly, CONFIRMED or SUSPECTED infection was not common in those cardiomyopathy and heart failure. We also note that infants and young children ( $<5$ years of age) had a lower prevalence of AKI than older children and young adults. While our exclusion of those hospitalized in Neonatal Intensive Care Units may have confounded these findings, this rate is lower than reported rates of AKI in other populations of septic neonates ${ }^{36,37}$. An ongoing study is currently reporting AKI prevalence in infants with suspected or confirmed SARS-CoV2 ${ }^{38}$ and should serve to expand and refine our findings in this age demographic. Further, we had more ability to show the relationships between age, ethnicity, and other chronic conditions in relation to both severity of illness in SARS-CoV2 and also with AKI in this setting.

Multi-inflammatory syndrome in children (MIS-C) is now recognized as a distinct manifestation of the virus ${ }^{21,39}$. This post-infectious dysregulated inflammatory response syndrome is under-represented in this cohort as it was designed and conducted in the early portion of the pandemic. As such, we were not able to delineate patients with MIS-C as we only required site investigators to note "CONFIRMED" as inclusive of both PCR and Ab testing. The inclusion of both populations, however, is relevant and important as we compare cardiogenic shock in the context of SARS-CoV2 and AKI. Further studies that evaluate the relationship between MIS-C and AKI are needed-thus far the data mirror findings of a high prevalence of severe $\mathrm{AKI}^{40}$. It is possible, that even in the absence of negative PCR testing, retrospective adjudication would result in increased prevalence of MIS-C and associated organ dysfunction ${ }^{41,42}$.

Our study has notable strengths and limitations. This report is taken from participating centers and thus should be placed in the context of the evolution of this pandemic. Our study was in the first three months of the pandemic-with a majority of the patients identified from centers located in the Region of the
Americas as defined by the World Health Organization. We limited our enrollment only to those suspected or confirmed to have SARS-CoV2 infection, thus it is quite possible selection bias is introduced and may actually affect our findings of both AKI prevalence and associations-and should be considered when comparing to the general pediatric ICU population. To this point, the evolution of the testing standards used likely impacted some of our results - as a majority of the participating centers adopted multiple tests for acute (PCR) and historical (Ab) based detection strategies. It was not possible to harmonize all the different tests utilized for comparison. It is possible providers were more likely to suspect SARS-CoV2 with a negative test result in more severely ill patients than those who were less severely ill. We also may not have captured all suspected patients due to the challenges of doing remote research during an active pandemic. For this reason, we limited the analysis of AKI severity as the detailed analysis of AKI severity would require the inclusion of duration, change over time, and other associated confounding factors related to AKI in individual patients-data we did not capture in a point-prevalence method. In statistical modeling, collinearity existed between diagnosis: shock-vasopressor use and between respiratory distress-invasive respiratory support; only vasopressor use and invasive respiratory support were included as these were viewed to be more objective. To prevent overfitting, country of location and admission diagnosis of metabolic derangements were dropped.

\section{CONCLUSIONS}

The SARS-CoV2 virus is associated with a higher than baseline prevalence of AKI in critically ill children. The prevalence and severity of AKI in the context of viral infection are similar to children with sepsis. As the first year of the COVID pandemic leads into the second year, the absence of a vaccine available for use across pediatric patients creates the potential for an ongoing 
pandemic. Future studies are needed to study the relationship of the virus and AKI, including understanding the pathobiology of infection and the severity of dysfunction.

\section{REFERENCES}

1. Joshi, K. et al. Cardiac dysfunction and shock in pediatric patients with covid-19. JACC Case Rep. 2, 1267-1270 (2020).

2. Soni, S. L. et al. Demographic \& clinical profile of patients with covid-19 at a tertiary care hospital in North India. Indian J. Med. Res. https://doi.org/10.4103/ ijmr.IJMR_2311_20 (2020).

3. Davies, P. et al. Intensive care admissions of children with paediatric inflammatory multisystem syndrome temporally associated with Sars-Cov-2 (Pims-Ts) in the UK: a multicentre observational study. Lancet Child Adolesc. Health 4, 669-677 (2020).

4. Oualha, M. et al. Severe and fatal forms of covid-19 in children. Arch. Pediatr. 27, 235-238 (2020).

5. Shekerdemian, L. S. et al. Characteristics and outcomes of children with coronavirus disease 2019 (covid-19) infection admitted to US and Canadian Pediatric Intensive Care Units. JAMA Pediatr. 174, 868-873 (2020)

6. Sachdeva, R. et al. The impact of coronavirus disease 2019 pandemic on U.S. And Canadian Picus. Pediatr. Crit. Care Med. 21, e643-e650 (2020).

7. Tagarro, A. et al. Screening and severity of coronavirus disease $2019>$ (covid-19) in children in Madrid, Spain. JAMA Pediatr. https://doi.org/10.1001/jamapediatrics.2020.1346 (2020).

8. Peng, S. et al. Early versus late acute kidney injury among patients with covid-19-A multicenter study from Wuhan, China. Nephrol. Dial. Transpl. 35, 2095-2102 (2020).

9. Wu, T. et al. Multi-organ dysfunction in patients with covid-19: a systematic review and meta-analysis. Aging Dis. 11, 874-894 (2020).

10. Lim, M. A. et al. Multiorgan failure with emphasis on acute kidney injury and severity of covid-19: systematic review and meta-analysis. Can. J. Kidney Health Dis. 7, 2054358120938573 (2020)

11. Huang, C. et al. Clinical features of patients infected with 2019 novel coronavirus in Wuhan, China. Lancet 395, 497-506 (2020).

12. Guan, W. J. et al. Clinical characteristics of coronavirus disease 2019 in China. N. Engl. J. Med. 382, 1708-1720 (2020).

13. Cheng, Y. et al. Kidney disease is associated with in-hospital death of patients with covid-19. Kidney Int. 97, 829-838 (2020).

14. Hirsch, J. S. et al. Acute kidney injury in patients hospitalized with covid-19. Kidney Int. 98, 209-218 (2020).

15. Gupta, S. et al. Aki treated with renal replacement therapy in critically III patients with covid-19. J. Am. Soc. Nephrol. 32, 161-176 (2021).

16. Chueh, T. I., Zheng, C. M., Hou, Y. C. \& Lu, K. C. Novel evidence of acute kidney injury in covid-19. J. Clin. Med. 9, 3547(2020).

17. Gagliardi, l. et al. Covid-19 and the kidney: from epidemiology to clinical practice. J. Clin. Med. 9, 2506 (2020)

18. Wang, X. et al. Be aware of acute kidney injury in critically ill children with covid19. Pediatr. Nephrol. 36, 163-169 (2021).

19. Raina, R., Chakraborty, R., Sethi, S. K. \& Bunchman, T. Kidney replacement therapy in covid-19 induced kidney failure and septic shock: a pediatric continuous renal replacement therapy [Pcrrt] position on emergency preparedness with resource allocation. Front. Pediatr. 8, 413 (2020).

20. Deep, A., Bansal, M. \& Ricci, Z. Acute kidney injury and special considerations during renal replacement therapy in children with coronavirus disease-19: perspective from the critical care nephrology section of the european society of paediatric and neonatal intensive care. Blood Purif. 50, 150-160 (2020).

21. Lee, M., Hilado, M., Sotelo, S., Opas, L. M. \& Im, D. D. Acute kidney injury in multisystem inflammatory syndrome in children (Mis-C): a case report. SN Compr. Clin. Med. https://doi.org/10.1007/s42399-020-00647-9 (2020).

22. Bjornstad, E. C. et al. Preliminary assessment of acute kidney injury in critically ill children associated with Sars-Cov-2 infection: a multicenter cross-sectional analysis. Clin. J. Am. Soc. Nephrol. 16, 446-448 (2020).

23. Dong, Y. et al. Epidemiological characteristics of 2143 pediatric patients with 2019 coronavirus disease in China. Pediatrics https://doi.org/10.1542/peds.2020-0702 (2020).

24. Harris, P. A. et al. The redcap consortium: building an international community of software platform partners. J. Biomed. Inf. 95, 103208 (2019).

25. Harris, P. A. et al. Research electronic data capture (redcap)-a metadata-driven methodology and workflow process for providing translational research informatics support. J. Biomed. Inf. 42, 377-381 (2009).

26. Green, D. A. et al. Clinical performance of Sars-Cov-2 molecular tests. J. Clin. Microbiol. 58, e00995-20 (2020).

27. Hoste, L. et al. A new equation to estimate the glomerular filtration rate in children, adolescents and young adults. Nephrol. Dial. Transpl. 29, 1082-1091 (2014).

28. Kaddourah, A., Basu, R. K., Bagshaw, S. M., Goldstein, S. L. \& Investigators, A. Epidemiology of acute kidney injury in critically ill children and young adults. $N$. Engl. J. Med. 376, 11-20 (2017).
29. Basu, R. K. et al. Clinical phenotypes of acute kidney injury are associated with unique outcomes in critically ill septic children. Pediatr. Res. https://doi.org/ 10.1038/s41390-021-01363-3 (2021).

30. Weiss, S. L. et al. Surviving sepsis campaign international guidelines for the management of septic shock and sepsis-associated organ dysfunction in children. Pediatr. Crit. Care Med. 21, e52-e106 (2020)

31. Weiss, S. L. et al. Global epidemiology of pediatric severe sepsis: the sepsis prevalence, outcomes, and therapies study. Am. J. Respir. Crit. Care Med. 191, 1147-1157 (2015)

32. Stewart, D. J. et al. Renal dysfunction in hospitalised children with covid-19. Lancet Child Adolesc. Health 4, e28-e29 (2020).

33. Qiu, H. et al. Clinical and epidemiological features of 36 children with coronavirus disease 2019 (covid-19) in Zhejiang, China: an observational cohort study. Lancet Infect. Dis. 20, 689-696 (2020).

34. Basu, R. K., Kaddourah, A., Goldstein, S. L. \& Investigators, A. S. Assessment of a renal Angina index for prediction of severe acute kidney injury in critically ill children: a multicentre, multinational, prospective observational study. Lancet Child Adolesc. Health 2, 112-120 (2018).

35. LaRovere, K. L. et al. Neurologic involvement in children and adolescents hospitalized in the United States for covid-19 or multisystem inflammatory syndrome. JAMA Neurol. 78, 536-547 (2021).

36. Jetton, J. G. et al. Incidence and outcomes of neonatal acute kidney injury (awaken): a multicentre, multinational, observational cohort study. Lancet Child Adolesc. Health 1, 184-194 (2017).

37. Starr, M. C. et al. The impact of increased awareness of acute kidney injury in the neonatal intensive care unit on acute kidney injury incidence and reporting: results of a retrospective cohort study. J. Perinatol. 40, 1301-1307 (2020).

38. Gale, C. et al. Characteristics and outcomes of neonatal Sars-Cov-2 infection in the UK: a prospective national cohort study using active surveillance. Lancet Child Adolesc. Health 5, 113-121 (2021).

39. Feldstein, L. R. et al. Multisystem inflammatory syndrome in U.S. children and adolescents. N. Engl. J. Med. 383, 334-346 (2020).

40. Deep, A. et al. Acute kidney injury in pediatric inflammatory multisystem syndrome temporally associated with severe acute respiratory syndrome coronavirus-2 pandemic: experience from Picus across United Kingdom. Crit. Care Med. 48, 1809-1818 (2020).

41. Alsaied, T. et al. Review of cardiac involvement in multisystem inflammatory syndrome in children. Circulation 143, 78-88 (2021).

42. Sperotto, F. et al. Cardiac manifestations in Sars-Cov-2-associated multisystem inflammatory syndrome in children: a comprehensive review and proposed clinical approach. Eur. J. Pediatr. 180, 307-322 (2021).

\section{ACKNOWLEDGEMENTS}

The principal investigators of the SPARC collaborative would like to acknowledge the research coordinators at each participating site for their contributions to this work (Supplemental File). The cloud-based data entry into Redcap is supported by a Center for Clinical and Translational Science and Training grant at the University of Cincinnati School of Medicine (2UL1TR001425-05A1).

\section{AUTHOR CONTRIBUTIONS}

R.K.B., E.C.B., K.A.K., M.Z., D.A., and S.L.G.: concept and design, data acquisition, analysis, interpretation, drafting, and final approval. K.M.G., M.S., P.K., and R.C.: interpretation, drafting, and final approval.

\section{COMPETING INTERESTS}

The authors declare no competing interests.

\section{ADDITIONAL INFORMATION}

Supplementary information The online version contains supplementary material available at https://doi.org/10.1038/s41390-021-01667-4

Correspondence and requests for materials should be addressed to R.K.B.

Reprints and permission information is available at http://www.nature.com/ reprints

Publisher's note Springer Nature remains neutral with regard to jurisdictional claims in published maps and institutional affiliations. 